\title{
Adjusted Sharpe Ratio: Some caveats
}

\author{
Didier MAILLARD ${ }^{1}$
}

November 2018

Acknowledgement: The author thanks Thierry Roncalli for valuable remarks and discussions.

\footnotetext{
${ }^{1}$ Professor (emeritus), Conservatoire national des arts et metiers, Senior Advisor, Amundi
} 


\begin{abstract}
Researchers and investors are concerned with the shortcomings of various measures of portfolio management performances, among them the famous Sharpe ratio. In particular, the Sharpe ratio does not give due consideration to tail risk: negative skewness and fat tails, which justly are a matter of concern for investors.

Various ways of correcting the Sharpe ratio have been proposed and continue to be proposed. One of them is the concept of Adjusted Sharpe Ratio (ASR) which gives a performance measure easy to compute from the basic statistics of returns.

The aim of this paper is to trace back the derivation of this formula and stress the assumptions and approximations needed for obtaining it. Those caveats should be kept in mind when using the ASR.
\end{abstract}

Keyword: Sharpe ratio, performance measures, tail risk

JEL Classification: C10, G11, G17 


\section{1 - Introduction}

Researchers and investors are concerned with the shortcomings of various measures of portfolio management performances, among them the famous Sharpe ratio. In particular, the Sharpe ratio does not give due consideration to tail risk: negative skewness and fat tails, which justly are a matter of concern for investors.

Various ways of correcting the Sharpe Ratio have been proposed and continue to be proposed (for a presentation see Maillard (2018)). One of them is the concept of adjusted Sharpe ratio (ASR) which gives a performance measure easy to compute from the basic statistics of returns.

$$
A S R=S R\left(1+\frac{S}{6} S R-\frac{(K-3)}{24} S R^{2}\right)
$$

As far as past performance is concerned, the Sharpe Ratio is easy to compute. $S$ is the skewness of the distribution of returns and $K$ its kurtosis. Those parameters are easy to compute from the third and fourth moments of the practical distribution.

Apparently, this simple and very manageable definition of an Adjusted Sharpe Ratio has been first proposed by Pézier (2004). It has been used in Pézier and White (2008) and by various authors, among then Brière \& al. (2015) and Kryzanowshi \& al. (2018). In Pézier (2011), an extension of the concept and a generalization are provided. However, we have not been able to recover the precise stuff of its derivation.

The aim of this paper is to trace back the derivation of this formula and stress the assumptions and approximations needed for its obtainment. Those caveats should be kept in mind when using the ASR. 


\section{2 - Derivation}

The derivation of the ASR requires five steps.

\section{Step 1: Certainty Equivalent Return (CER)}

The first step is to tie the performance measure to the supposed investor's utility function, which appears judicious. Investment will transform an initial wealth $w_{0}$ into an end-of-period wealth $w$. This final wealth will provide the investor with an utility $u(w)$. As the investment may be risky, the investor's satisfaction will be gauged, à la Neumann-Morgenstern, by its expectation $E[u(w)]$.

If the period's return is $r$, the expected utility will write $E\left(u\left(w_{0}(1+r)\right)\right)$. The Certainty Equivalent Return $c$ will be defined as the return on a risk-free asset needed to provide the same expected utility than the risky asset. In the case of the certainty equivalent return, utility and expected utility coincide.

$$
u\left(w_{0}(1+c)\right)=E\left[u\left(w_{0}(1+r)\right]\right.
$$

The CER is itself a performance measure taking risk into account.

\section{Step 2: Introducing a Constant Absolute Risk Aversion (CARA) utility function}

The investor's preferences are assumed to be represented by a CARA (otherwise named exponential) utility function:

$$
u(w)=-e^{-\frac{w}{\lambda}}
$$

where $1 / \lambda$ is a risk aversion parameter. By assuming that initial wealth is normalized to unity, we obtain:

$$
u(w)=-e^{-\frac{w_{0}(1+r)}{\lambda}}=-e^{-\frac{1+r}{\lambda}}=-e^{-\frac{1}{\lambda}} e^{-\frac{r}{\lambda}}
$$


It is possible to drop the constant term and write finally the utility function as:

$$
u(r)=-e^{-\frac{r}{\lambda}}
$$

\section{Step 3: Certainty Equivalent Return when return is Gaussian}

It also alleviates the notations to consider $r$ as representing the excess return above the riskfree rate (the risk-free component will be constant in the utility function). One can write:

$$
r=\mu+\sigma \varepsilon \quad E(\varepsilon)=0 \quad E\left(\varepsilon^{2}\right)=1
$$

with $\mu$ the expected (excess) return and $\sigma$ the return's volatility.

If returns are Gaussian, expected utility will write:

$$
E[u(r)]=-E\left[e^{-\frac{r}{\lambda}}\right]=-e^{-\frac{\mu}{\lambda}} E\left[e^{-\frac{\sigma \varepsilon}{\lambda}}\right]=-e^{-\frac{\mu}{\lambda}} e^{\frac{\sigma^{2}}{2 \lambda^{2}}}
$$

Since we have:

$$
u(c)=-e^{-\frac{c}{\lambda}}=E[u(r)]=-e^{-\frac{\mu}{\lambda}} e^{\frac{\sigma^{2}}{2 \lambda^{2}}}
$$

the CER $c$ is equal to:

$$
c=\mu-\frac{\sigma^{2}}{2 \lambda}
$$

\section{Step 4: Optimal investment}

This step is essential. The above CER corresponds to a full investment in the risky asset or risky portfolio under consideration. If a share $\omega$ (which may exceed 100\%) of wealth is invested in the risky asset, the rest in the risk-free asset, the portfolio's CER would write: 


$$
c_{\omega}=\omega \mu-\omega^{2} \frac{\sigma^{2}}{2 \lambda}
$$

which is not proportionate to the share invested in the risky asset but is first increasing and then decreasing.

From the point view of maximising the expected utility (and thus the CER), there is thus an optimal investment share in the risky asset. This optimal share is given by equating to zero the first derivative:

$$
\omega^{*}=\frac{\lambda \mu}{\sigma^{2}}
$$

We deduce that:

$$
c_{\omega}^{*}=c^{*}=\frac{\lambda \mu^{2}}{\sigma^{2}}-\frac{\lambda^{2} \mu^{2}}{\sigma^{4}} \frac{\sigma^{2}}{2 \lambda}=\frac{\lambda^{2} \mu^{2}}{2 \sigma^{2}}=\frac{\lambda}{2} s^{2}
$$

where $\mathrm{s}=\mu / \sigma$ is the Sharpe Ratio.

There is thus, in the Gaussian case, a relationship between the Sharpe ratio and the certainty equivalent return.

$$
s=\sqrt{2 c^{*} / \lambda}
$$

\section{Step 5: Generalizing to non-Gaussian returns}

The next step will be to compute a CER for non Gaussian returns and define an adjusted Sharpe ratio by:

$$
s_{a}=\sqrt{2 c^{*} / \lambda}
$$

One important assumption at this stage is that the optimal portfolio, composed of the risky asset or portfolio and the risk-free asset, that is used for the computation of the CER will have the same weights as the optimal portfolio in the case of Gaussian returns 
of same mean and volatility. This means that it is not really optimal in the non-Gaussian case...

Since $\omega^{*}=\frac{\lambda \mu}{\sigma^{2}}$, we notice that this "optimal" portfolio has mean and volatility:

$$
\mu^{*}=\omega^{*} \mu=\lambda s^{2} \quad \sigma^{*}=\omega^{*} \sigma=\lambda s
$$

The "optimal" CER will verify:

$$
u\left(c^{*}\right)=E[u(r)]=-e^{-\frac{\mu *}{\lambda}} E\left[e^{-\frac{\sigma * \epsilon}{\lambda}}\right]
$$

Contrarily to the Gaussian case, there is not an analytical result for the above expected value. The method is to replace the utility function by a fourth-order Taylor expansion around the mean return of the optimal portfolio $\mu^{*}$.

$$
u(r) \cong u+u^{\prime}\left(r-\mu^{*}\right)+\frac{1}{2} u^{\prime \prime}\left(r-\mu^{*}\right)^{2}+\frac{1}{6} u^{\prime \prime \prime}\left(r-\mu^{*}\right)^{3}+\frac{1}{24} u^{\prime \prime \prime}\left(r-\mu^{*}\right)^{4}
$$

The derivatives of the CARA utility function are:

$$
u^{\prime}=-\frac{1}{\lambda} u \quad u^{\prime \prime}=\frac{1}{\lambda^{2}} u \quad u^{\prime \prime \prime}=-\frac{1}{\lambda^{3}} u \quad u^{\prime \prime \prime}=\frac{1}{\lambda^{4}} u
$$

while the first moments of the returns are:

$$
E\left(r-\mu^{*}\right)=0 \quad E\left(\left(r-\mu^{*}\right)^{2}\right)=\sigma^{* 2} \quad E\left(\left(r-\mu^{*}\right)^{3}\right)=S \sigma^{* 3} \quad E\left(\left(r-\mu^{*}\right)^{4}\right)=K \sigma^{4}
$$

We deduce that:

$$
E[u(r)] \cong u\left[1+\frac{1}{2} \frac{1}{\lambda^{2}} \sigma^{* 2}-\frac{1}{6} \frac{1}{\lambda^{3}} S \sigma^{* 3}+\frac{1}{24} \frac{1}{\lambda^{4}} K \sigma^{* 4}\right]
$$

Finally, we obtain that:

$$
E[u(r)] \cong u\left[1+\frac{1}{2} s^{2}-\frac{1}{6} S s^{3}+\frac{1}{24} K s^{4}\right]
$$


On the other side of the equation, a one-order Taylor development is considered as sufficient, so that:

$$
u\left(c^{*}\right) \cong u+u^{\prime}\left(c^{*}-\mu^{*}\right)=u\left[1-\frac{1}{\lambda}\left(c^{*}-\mu^{*}\right)\right]=u\left[1+s^{2}-\frac{1}{\lambda} c^{*}\right]
$$

as $\mu^{*}=\lambda s^{2}$

The equality between expected utility and utility of the certainty equivalent return yields:

$$
\begin{gathered}
1+s^{2}-\frac{1}{\lambda} c^{*} \cong 1+\frac{1}{2} s^{2}-\frac{1}{6} S s^{3}+\frac{1}{24} K s^{4} \\
\frac{1}{\lambda} c^{*} \cong \frac{1}{2} s^{2}+\frac{1}{6} S s^{3}-\frac{1}{24} K s^{4}
\end{gathered}
$$

As may be seen in the Gaussian case $(S=0, K=3)$, these developments give an approximation: the CER is decreased by a fourth-order term. However, the suggestion is to use the Taylor developments not to compute the CER, but the difference between the CER and the exact CER in the Gaussian case. This will lead to:

$$
c^{*}=\frac{\lambda}{2} s_{a}^{2} \cong \frac{\lambda}{2} s^{2}+\frac{\lambda}{6} S s^{3}-\frac{\lambda}{24}(K-3) s^{4}=\frac{\lambda}{2} s^{2}\left[1+\frac{\lambda}{3} S s-\frac{\lambda}{12}(K-3) s^{2}\right]
$$

or:

$$
s_{a} \cong s\left[1+\frac{\lambda}{3} S s-\frac{\lambda}{12}(K-3) s^{2}\right]^{1 / 2}
$$

The ultimate step is to approximate this radical, leading to:

$$
s_{a} \cong s\left[1+\frac{\lambda}{6} S s-\frac{\lambda}{24}(K-3) s^{2}\right]
$$




\section{3 - A link to Aumann \& Serrano's Economic Index of Riskiness}

Aumann and Serrano (2008) have proposed an economic index of riskiness. For a gamble or a lottery whose pay-off is a random variable $g$, the economic index of riskiness $R$ is defined as:

$$
E\left[e^{-g / R}\right]=1
$$

Applied to investment (Maillard, 2018), the gamble may be interpreted as the swap between the risk-free rate and the return of a risky asset or portfolio. If $r$ is the excess return of the risky asset or portfolio, the economic index of riskiness will verify:

$$
E\left[e^{-r / R}\right]=1
$$

On the left-hand side, we recognize a CARA expected utility with absolute risk aversion parameter equal to $1 / R$. On the right-hand side of the equation, we may recognize the utility, with the same utility function, of a Certainty Equivalent Return equal to zero.

Aumann and Serrano's economic index of riskiness may actually be interpreted as the threshold, in terms of risk aversion, at which the risky asset or portfolio's return is equivalent to the risk-free rate. 


\section{References}

Aumann, R. J. and R. Serrano, 2008, “An Economic Index of Riskiness”, Journal of Political Economy, vol. 116(5), pp. 810-836

Brière, Marie, K. Oosterlinck, A. Szafarz, 2015, "Virtual currency, tangible return: Portfolio diversification with bitcoin", Journal of Asset Management, November 2015, vol. 16, issue 6, pp 365-373

Kryzanowski, L., Jie Zhang and Rui Zhonga "Currency Hedging of International Bond Portfolios: Effects of Unconventional Monetary Policy Regimes », SSRN Working Paper, \# 3123960

Maillard, D., 2018, "Tail Risk Adjusted Sharpe Ratio", Amundi Working Paper WP-071, available on Amundi Research Center

Pézier, Jacques, 2004, "Risk and Risk Aversion", in C. Alexander and E. Sheedy, eds.: The Professional Risk Managers'Handbook, PRMIA Publications

Pézier, J. and A. White, 2008, “The relative merits of alternative investments in passive portfolios, Journal of Alternative Investment 10(4), pp. 37-49

Pézier, J., 2011, "Rationalization of Investment Preference Criteria", ICMA Discussion Papers in Finance, DP2011-12 